\title{
Discrepant Responding Across Measures of College Students' Sexual Victimization Experiences: Conceptual Replication and Extension
}

\author{
RaeAnn E. Anderson \\ University of North Dakota, raeann.anderson@UND.edu \\ Douglas D. Delahanty
}

\section{How does access to this work benefit you? Let us know!}

Follow this and additional works at: https://commons.und.edu/psych-fac

Part of the Psychology Commons

\section{Recommended Citation}

RaeAnn E. Anderson and Douglas D. Delahanty. "Discrepant Responding Across Measures of College Students' Sexual Victimization Experiences: Conceptual Replication and Extension" (2019). Psychology Faculty Publications. 23.

https://commons.und.edu/psych-fac/23

This Article is brought to you for free and open access by the Department of Psychology at UND Scholarly Commons. It has been accepted for inclusion in Psychology Faculty Publications by an authorized administrator of UND Scholarly Commons. For more information, please contact und.commons@library.und.edu. 
Discrepant Responding Across Measures of College Students’ Sexual Victimization

Experiences: Conceptual Replication and Extension

First submission: January $25^{\text {th }}, 2019$

Revision submitted: June $20^{\text {th }}$, 2019

Second revision submitted: September $4^{\text {th }}$, 2019 


\begin{abstract}
Sexual violence victimization affects approximately 1 in 5 college women and 1 in 6 college men; however, rates of sexual victimization vary widely, in part due to measurement issues. The present study is the first to compare the Sexual Experience Survey-Short Form Victimization to a measure of sexual victimization designed to capture gender differences, the Post-Refusal Sexual Persistence Scale-Victimization (PRSPS-V). Prior research has compared the perpetration versions of these questionnaires and found large discrepancies. College students $(\mathrm{N}=673: 367$ women, 298 men, 8 gender minority) were surveyed. The SES-SFV identified 260 cases of sexual victimization whereas the PRSPS-V identified 330 cases; this discrepancy was largest for men. While percent agreement between the two measures ranged from 79.9-92.0\%, kappa estimates indicated that agreement was in the weak to moderate range. Kappa estimates tended to be poorer for men than women. These results highlight poor precision in the measurement of sexual violence victimization, even when using well-established measures. The PRSPS-V identified more cases and may be less gender biased. We discuss how differences in questionnaire structure, item structure, and operationalization of consent may account for discordance between the SES-SFV and PRSPS-V even when controlling for item content. Keywords: sexual violence, rape, assessment, measurement, coercion
\end{abstract}


Discrepant Responding Across Measures of College Students’ Sexual Victimization Experiences: Conceptual Replication and Extension

Rape affects approximately 1 in 5 college women (Post, Biroscak, \& Barboza, 2011; Muehlenhard, Peterson, Humphreys, \& Jozkowski, 2017) and 1 in 6 college men (Anderson, Cahill, \& Delahanty, 2018a). However, the exact scope of sexual violence (including rape), is uncertain among less studied populations due, in part, to measurement issues. For example, estimates of sexual violence victimization in men range from 2 to $73 \%$ depending on the definition and instrument used (Peterson, Voller, Polusny, \& Murdoch, 2010). Research that can elucidate the strategies necessary to obtain accurate estimates of sexual violence is greatly needed in order to understand the scope of this problem and improve prevention and treatment efforts across populations. For example, Strang and colleagues compared two strategies for assessing sexual perpetration, The Sexual Experiences Survey - Short Form Perpetration (SESSFP) and the Sexual Strategies Scale (SSS) and identified several important characteristics of the questionnaires that may influence their psychometric properties (Strang, Peterson, Hill \& Heiman, 2013; Strang \& Peterson, 2017). The goal of this study was to conceptually replicate Strang et al., (2013) by comparing two different strategies for assessing sexual violence victimization histories, The Sexual Experiences Survey - Short Form Victimization (SES-SFV) and The Post-Refusal Persistence Scale - Victimization (PRSPS-V), with male and female college students. This study extends Strang et al., (2013) by testing some of the hypothesized mechanisms for the sources of discrepancies in their work on sexual perpetration in the realm of sexual victimization and by examining women as well as men.

\section{Struggles with Imprecision in Sexual Violence Measurement}

Sexual violence is any form of sexual contact that occurs without consent (Basile, Smith, Breiding, Black \& Mahendra, 2014) and can take several forms which range in severity. Rape, or 
the use of force, substance intoxication, or threats of force to coerce sex is considered the most severe form (Koss et al., 2007). Although terminology varies, in this paper we will use the term sexual victimization to refer to the experiences of people who have been harmed by sexual violence and sexual perpetration to refer to the experiences of people whose behavior harms others sexually.

There have been several calls to improve measurement tools in violence research, including ones specific to sexual violence (Teten Tharp et al., 2013; Krahé \& Vanwesenbeeck, 2015). Methodological variation in measurement undoubtedly contributes to confusion over the utility and strength of risk factors for sexual violence perpetration (Bouffard \& Goodson, 2017), and perhaps mis-estimates of the efficacy of prevention programs. This imprecision is exacerbated when sexual victimization is examined in less well-studied populations - such as men. Prior research comparing measures of sexual violence in men has suggested that some discordance between measures is driven by varying degrees of gendered assumptions inherent in some measures (Anderson, Cahill, \& Delahanty, 2018b). Indeed, most of the currently available measures were designed to assess sexual victimization in women and were later adapted for men rather than including men during their development. For example, current SES-SFV items do not assess men (and other people with penises) being forced to penetrate another person's vagina. Similarly, research on men's victimization is usually conducted separately from research with women; however, direct comparison of men’s and women's experiences can be helpful particularly for ascertaining the degree and type of gender differences in sexual violence. To wit, Buday \& Peterson (2015) demonstrated how some college women, but not men, interpreted the SES perpetration items as cues to disclose experiences of sexual victimization, the opposite of the intended meaning. Better understanding of these gender differences can lead to 
improvements in measuring the prevalence and therefore understanding the scope of sexual victimization.

\section{Development and Use of the Sexual Experiences Surveys}

The original (1982) Sexual Experiences Survey (SES) was developed in a college population with the goal of assessing sexual victimization from a dimensional perspective and capturing incidents that met the legal definition of a crime but were underreported to law enforcement (Koss \& Oros, 1982). Notably, a simultaneous perpetration version of the 1982 SES was developed by changing the pronouns in the heterosexual, cisgender, woman-centered victimization version; a strategy now considered less than optimal as this conflates the victim's experience with the perpetrator's and presumes heterosexuality (Koss et al., 2007). The psychometric properties of the 1982 SES were extensively researched. Multiple studies assessed test-retest reliability (Cecil \& Matson, 2006; Koss \& Gidycz, 1985; Krahé, Reimer, Scheinberger-Olwig, \& Fritsche, 1999) and construct validity (Karabatsos, 1997; Koss \& Gidycz, 1985; Testa, VanZile-Tamsen, Livingston, \& Koss, 2004). However, these studies were almost exclusively conducted with heterosexual women. The SES has since undergone two revisions, one in 1987 and one in 2007. The 2007 revision focused on reducing gender bias, reducing heterosexism, and broadening the range of sexual victimization experiences assessed (Koss et al., 2007). The result was the SES-Short and -Long Form Victimization scales (SESSFV and SES-LFV, respectively) as well as paired versions assessing sexual perpetration.

The SES-SFV has 35 items compared to the 12 items of the 1982 SES, reflecting the much more comprehensive and detailed nature of the SES-SFV. Each SES-SFV item is a pairing of a sexual outcome such as, “someone had oral sex with me or made me have oral sex...” with a tactic that may have been used to coerce that behavior such as, "by taking advantage of me when 
I was too drunk or out of it to stop what was happening”. Since 2007, the SES-SFV has become widely used (cited over 700 times in Google Scholar as of June 2019). Research has provided good evidence for the validity of the SES-SFV with young community and college women (Davis et al., 2014; Johnson, Murphy \& Gidycz, 2017), adequate evidence of validity with college men (Anderson et al., 2018a), but poor to adequate evidence of reliability with college men (Anderson et al., 2018a; Johnson et al., 2017). Research on other sexual victimization questionnaires in men has also documented poorer evidence of validity when comparing correlation values to those obtained with samples of women (French, Suh, \& Arterberry, 2017).

\section{Development of the Post-Refusal Sexual Persistence Scales}

In contrast, the Post-Refusal Sexual Persistence Scale-Victimization (PRSPS-V) was developed specifically to assess gender differences in sexual victimization experiences and thus, included male participants during the development of the measure. Further, the PRSPS-V was crafted based on reviewing and synthesizing several existing measures, including the 1982 SES, and therefore benefitted from a larger pool of available literature at the time of its development (Struckman-Johnson, Struckman-Johnson, \& Anderson, 2003). Given these differences in development, the PRSPS-V may have greater validity for detecting sexual victimization in men. However, less attention has been paid to the psychometric properties of the PRSPS-V than the SES-SFV. We are unaware of any test-retest reliability data for the PRSPS-V. StruckmanJohnson, Struckman-Johnson \& Anderson (2003) provided some evidence for the construct validity of the PRSPS-V with college students (men and women) by asking participants who endorsed a PRSPS-V item to describe the most recent experience of unwanted sexual contact related to that item. Most participants (82\%) provided a written description of an incident consistent with sexual victimization. 


\section{Comparing Available Measurement Strategies}

Comparing two measures of the same construct can help identify and clarify when and why research demonstrates different prevalence rates for similar (or the same) behaviors (Cascardi \& Muzyczyn, 2016; DiLillo et al., 2006; Strang et al., 2013). Strang and colleagues highlighted discrepancies between the perpetration version of the SES-SFV (the SES-SFP) and a modified PRSPS-Perpetration (the Sexual Strategies Scale: the SSS) across multiple studies (Strang et al., 2013; Strang \& Peterson 2017). Specifically comparing the SES-SFP to the SSS, they found that percent agreement in identifying any cases of sexual perpetration was $60.6 \%$, kappa $=.25$. This degree of kappa indicates minimal agreement between the two independent "raters," or in this case, questionnaires, such that $6.3 \%$ of the data would be considered reliably agreed upon regarding sexual victimization status; this is well below suggested clinical guidelines (McHugh, 2012).

Strang et al., (2013)’s work highlighted multiple possible explanations for these discrepancies, particularly highlighting differences in the operationalization of consent, and both questionnaire and item structure. These findings are particularly notable in that the SES-SFP and the SSS assess the same construct, and these discrepancies remained even when Strang et al., (2013) controlled for item content. However, Strang and colleagues note that their results may have been influenced by the aforementioned structural differences between the questionnaires as well as methodological issues such as administrating the questionnaires in a fixed order. Other research has demonstrated discrepancies between the 1982 SES and the Revised Conflict Tactics Scale (CTS2: Cook, 2002), the SES-SFV and the CTS2-SC (Moreau, Boucher, Hébert \& Lemelin, 2014; Anderson et al., 2018b), and the SES-SFP and the CTS2 (Anderson et al., 
2018b). Thus, issues in measurement imprecision are not unique to the SES-SFP and the SSS and likely apply to the SES-SFV and the PRSPS-V.

\section{Current Study: Conceptual Replication and Extension}

Given prior research documenting discrepancies between measures of sexual perpetration and gender differences in the psychometric properties of sexual victimization measures, more research on sexual violence victimization measurement is needed that provides clarity on good measurement practices. To our knowledge, no prior research has compared the PRSPS-V to the SES-SFV in terms of concordance (agreement between the two measures); yet, the PRSPS-V appears to be a promising tool that may be less gender-biased than the SES-SFV.

The current study compared the prevalence rates of sexual victimization in male and female college students as assessed by the SES-SFV and the PRSPS-V as a conceptual replication and extension of Strang et al., (2013). Although Strang et al., (2013) focused on sexual perpetration in men due to the dearth of research on measurement practices in sexual perpetration research, sexual victimization in men is even more under-researched. Further, the issues raised by Strang et al., (2013) regarding the perpetration versions of the SES-SFV and the PRSPS-V are likely also applicable to the victimization versions of these questionnaires. The SES-SFV/SES-SFP and the PRSPS-V/SSS were specifically designed to mirror one another. Thus we seek to conceptually replicate Strang et al. (2013)’s findings with a focus on victimization rather than perpetration. In contrast to Strang et al., we recruited a large sample of female and male college students to assess potential gender differences in concordance (or lack thereof). We also attempted to control for methodological issues raised by Strang and colleagues by utilizing the same response format across the questionnaires, adding items to equalize item content, and counterbalancing the order of administration of the questionnaires. We further 
report kappa estimates as a measure of the degree of agreement between the two questionnaires (analogous in this instance to independent raters) that controls for chance agreement rather than solely relying on percent agreement.

Hypotheses:

1. The PRSPS-V will identify more cases of sexual violence victimization than the SESSFV (Hypothesis 1a), even when controlling for item content (Hypothesis 1b: Strang et al., 2013; Strang \& Peterson, 2017) across genders consistent with Strang et al. (2013)'s findings of higher prevalence rates for the SSS (a modification of the PRSPS-perpetration) compared to the SES-SFP.

2. Further, we will examine and quantify concordance for specific tactics of sexual victimization including verbal coercion (Hypothesis 2a), alcohol-facilitated coercion (Hypothesis 2b), and physical force coercion (Hypothesis 2c). We expect concordance will be the worst for verbal coercion given prior research highlighting discordance for this tactic (Anderson et al., 2018b; Strang et al. 2013). We expect that kappa values will be largely in the acceptable range (weak to moderate agreement) rather than in the strong range (strong or nearly perfect agreement) for every type of victimization compared (Hypotheses 1a-2c). We will also investigate gender differences in concordance although we consider these analyses exploratory.

3. There will be no effect for order of administration on patterns of discordance given prior research (Anderson et al., 2018b). 


\section{Method}

\section{Participants}

Participants were 673 college students at a large, public, Midwestern university with a mean age of 19.5 (SD 3.6). Women comprised 54.5\% $(n=367)$ of the sample, men - 44.3\% $(n=$ 298), and transgender and other gender identities - 1.1\% ( $n=8$ gender minorities). Most participants reported being heterosexual (85.6\%); some were bisexual (5.6\%), gay (4.9\%), and queer (1.5\%). A small number of participants reported their sexual orientation as other $(n=31)$; on reviewing the descriptive labels provided by participants a minority of these participants were re-classified as heterosexual because they provided self-labels consistent with heterosexuality (e.g., "regular” or “straight”). Participants were mostly Caucasian (85.6\%); some were African American (9.4\%), Asian American (3.7\%), and Native American (1.0\%). A few participants reported identifying as Latinx (3.7\%). The average income of participants' families was in the $\$ 60,000-79,999$ range.

\section{Measures}

\section{Questionnaires assessing sexual violence victimization.}

The Sexual Experiences Survey - Short Form Victimization (SES-SFV: Koss et al., 2007). The original version of the SES-SFV consists of five items for men and seven items for women. All of these are compound, behaviorally-specific items that begin with a stem describing a sexual behavior (i.e., “someone forced me to perform oral sex...”) followed by five possible tactics that were used to coerce the sexual behavior (verbal criticism, verbal pressure, alcohol incapacitation, threats of physical force, physical force). This creates 25-35 items by crossing each sexual act by each tactic (see Table 1 for an example item); items are ordered hierarchically starting with less severe items and progressing to more severe items. The SES-SFV has demonstrated evidence of 
good convergent validity and test-retest reliability in past research with college women (Johnson, Murphy, \& Gidycz, 2017). It has also demonstrated good evidence of convergent validity with college men and adequate evidence of test-retest reliability when scored dichotomously but poorer evidence of test-retest reliability when scored categorically/ordinally (Anderson et al., 2018a). Following, for this study, we utilized a dichotomous response format (yes or no) to assess whether each experience had ever occurred since age 14.

Changes made to the SES-SFV. Given our interest in gender differences in the current study, we modified the SES-SFV to be more gender neutral and inclusive following Hipp \& Cook (2017)’s suggestions for research and evidence from Anthony and Cook (2012). For example, item 4 on the SES-SFV assessed completed vaginal penetration but only for women. However, it is possible for a male to be forced to penetrate a woman's vagina with his penis or a woman to be forced to penetrate a vagina with fingers or objects; thus, we added three items designed to more broadly capture possible experiences of being forced to engage in vaginal or anal sex. For example, “A woman MADE ME put my penis into her vagina, or MADE ME insert my fingers or objects into her vagina without my consent” assesses completed forced penetration of a vagina for both men and women. Similarly, "Someone MADE ME put my penis into their butt, or someone MADE ME insert my fingers or objects without my consent” assesses forced penetration of the anus. For example, a person may be so incapacitated by alcohol that they are able to be coerced into putting their penis or fingers into someone else’s butt. Finally, “Even though it didn't happen, a man tried to MAKE ME put his penis into my butt, or someone tried to MAKE ME stick in objects or fingers without my consent.” These three additional items were added for all genders such that our revised version of the SES-SFV contained 50 items for women (10 sexual behaviors x 5 tactics) and 40 items for men (8 sexual behaviors $\mathrm{x} 5$ tactics). 
All participants were presented with all items; however, an additional instruction preceded the vaginal penetration and attempted vaginal penetration items, "skip this item if you do not have a vagina” to direct male-identifying participants to the remaining items.

The Post-Refusal Sexual Persistence Scale (PRSPS-V: Struckman-Johnson, StruckmanJohnson, \& Anderson, 2003). The PRSPS-V contains 19 items that assess sexual victimization in relation to five different types of tactics: enticement, verbal coercion, misuse of authority, alcohol/drugs, and physical force (see Table 1 for example items). In contrast to the SES-SFV, there is an initial instruction set to the PRSPS-V that defines sexual contact as "genital touching, oral sex, or intercourse” and then gives a list of 19 ways in which sexual contact may have been coerced such as "someone threatened me with a weapon.” Thus, PRSPS-V items are not compound and are only behaviorally specific regarding the tactic; further, these items are administered in a randomized order. The original PRSPS-V then asks participants to write the number of times they experienced that tactic next to the item. The PRSPS-V is appropriate for any gender and has demonstrated good evidence of convergent validity in prior research with college women and men (Struckman-Johnson et al., 2003); PRSPS-V scores are correlated with increased substance use and sexual dysfunction (Turchik \& Hassija, 2014). We were unable to locate any estimates of test-retest reliability for the PRSPS-V.

Changes made to the PRSPS- $V$. We made some modifications to the PRSPS-V to make it more comparable to the SES-SFV. For instance, we used a dichotomous response format rather than eliciting frequencies. We further defined sexual contact by adding "kissed, fondled...anal sex" to the instructions as the SES-SFV assesses these types of sexual contact. We also revised the instructions to be more gender neutral using “their” instead of "his” in describing nonconsent in some items, for example, "They took advantage of the fact that you were drunk or 
high" instead of using "He" as the noun describing the perpetrator. We also changed the timeframe to any incidents that occurred since age 14 rather than age 16 to be equivalent to the timeframe of the SES-SFV. These changes are also consistent with those made by Strang and colleagues (Strang et al., 2013).

Scoring. Given the goals of the study to focus on concordance and possible areas of discrepancy we used dichotomous scores for both the SES-SFV and the PRSPS-V. Participants who responded "yes" to any item on either the SES-SFV or the PRSPS-V were coded as having a history of sexual victimization. We also calculated sexual victimization by tactic regardless of the sexual outcome: verbal coercion, alcohol or drug incapacitation, and physical force/threats of force. Table 1 describes and compares/contrasts all SES-SFV and PRSPS-V items. We did not compute Cronbach's alpha for either measure of sexual violence victimization as Cronbach's alpha is a measure of reliability recommended for latent constructs wherein each item is presumed to represent a facet of the latent construct. The SES-SFV and the PRSPS-V are measures of behavioral experiences rather than latent constructs; there is no hypothesized latent construct within participants that is presumed to cause sexual victimization nor are individual experiences of victimization necessarily related to one another (Diamantopoulos, Riefler, \& Roth, 2008; Koss et al., 2007).

\section{Procedures}

The Institutional Review Board of the second author approved the following procedures. Data were collected between September-December 2017 for an online study advertised as “Questionnaires about Sexual Behavior.” Data were collected anonymously. Participants completed the study at the time of their choosing and received credit using the Sona experiment management system. The survey was administered outside of Sona using Qualtrics. Participants 
completed consent and a demographics form and then the remaining study questionnaires were administered in a randomized order. The present study was part of a larger research study examining the validity of sexual victimization questionnaires. Additional measures (of rape empathy and understanding of consent) beyond the scope of this paper were also administered.

\section{Results}

\section{Data cleaning, analytic plan, and power analysis}

Any participant who completed at least one item on the SES-SFV and the PRSPS-V was included; 11 did not complete any SES-SFV or PRSPS-V items and were excluded, leaving 673 participants. If a participant did not complete an item on the sexual victimization questionnaires, their response to that item was assumed to be the modal response, zero. Missing data for most SES-SFV items was very minimal; on average each item had missing data for only one respondent. However, the amount of missing data for the anal sex and forced penetration items was higher, $n \approx 40-120$. Analysis of the forced penetration items indicates that most of the participants who skipped these items were women (approximately 90\%) - thus their skipping of these items was likely appropriate ${ }^{1}$ given the extremely low base rate of forced anal penetration for women (e.g., a women being forced to put her fingers or an object in someone’s anus). Missing data for PRSPS-V items was $n \approx 2$. At this time, data are available upon request from the corresponding author and are not yet publicly available in order to respect the rights of research participants who did not consent to their data being shared publicly. The entire sample (including sexual and gender minorities) was used to make overall comparisons. In follow-up analyses examining gender differences, the women only $(n=376)$ and men only $(n=298)$

\footnotetext{
${ }^{1}$ Eight men completed less than $67 \%$ of these items; when we re-ran analyses excluding these men the percentage agreement changed marginally $(<.4 \%)$. We also re-ran analyses excluding any participant who had any missing data and results were consistent with those presented here.
} 
groups were used; these groups were determined by participant self-identification from a single item.

According to a priori power analyses, we had a sufficient total sample size to detect most small effects $(w=.15$, suggested $n=349$ for Power $=.80)$ for chi-square analyses. However, post-hoc power analyses suggest we did not have an adequate sample size to detect small effect sizes $(w=.1)$ for sexual orientation differences (suggested $n=191$ for Power $=.80$ ). Thus, we only report prevalence rates for sexual minorities rather than concordance.

\section{Descriptive Results}

Considering cases identified by either measure, the prevalence rate of sexual victimization was $53.2 \%$ for the entire sample. A majority (64.3\%) of 376 women respondents reported at least one incident of sexual victimization, while 38.9\% of 298 men respondents and $100 \%$ of eight gender minority respondents reported at least one incident of sexual victimization of any type. When considering the interaction of sexual orientation $\mathrm{x}$ gender victimization, rates were: sexual minority women $-62.2 \%$ of $n=45$, sexual minority men $-46.7 \%$ of $n=45$, heterosexual women $-64.6 \%$ of $n=322$, heterosexual men $-37.5 \%$ of $n=253$.

\section{Hypothesis 1: Questionnaire Differences in Victimization Rates}

We next computed cross-tabulations and chi-squares to determine whether the different questionnaires resulted in different rates of victimization reporting. We calculated percent agreement to reflect concordance between the measures. Discordance (e.g., discrepancy) is the inverse of percent agreement - computed by dividing the number of cases where the questionnaires disagree (SES-SFV no/PRSPS-V yes or SES-SFV yes/PRSPS-V no) by the total number of cases (673). We used McHugh (2012)'s guidelines for interpreting kappa which 
suggest a minimum of kappa $=.80$ for health research. The following analyses are also presented in Table 2.

Overall victimization rates (H1a). The prevalence rate for any sexual victimization was $38.6 \%$ (51.5\% of women, $21.8 \%$ of men) for the SES-SFV and was $49.0 \%$ for the PRSPS-V (59.1\% of women, $35.6 \%$ of men). We compared all SES-SFV items to the 19 PRSPS-V items. The SES-SFV identified 260 participants while the PRSPS-V identified 330 participants. The SES-SFV identified 30 participants that the PRSPS-V did not; the PRSPS-V identified 100 participants that the SES-SFV did not for a total of 130 discordant cases. Percent agreement was 80.7\% (i.e., 543/673) for lifetime sexual victimization. The rate of discordance was statistically significant, $\chi(1)=263.55, p<.001$, indicating that the questionnaires identified a different number of individuals reporting lifetime sexual victimization. Kappa was .61, indicating moderate agreement when adjusting for chance. The effect size for this concordance was Phi = .63, correspondingly, the effect size for discordance would be Phi = .37 (e.g., 1.0 minus .63); Phi of .37 is considered a medium effect size. When testing for gender differences, the PRSPS-V detected significantly more cases of sexual victimization than the SES-SFV for both men (35.6\% vs. $21.8 \%)$ and women $(59.1 \%$ vs. $51.5 \%), \chi^{2}(1)>4.39, p<.04$. Percent agreement was not significantly different for women (81.7\%) than for men (79.5\%). However, kappa values did fall into different interpretative categories, kappa = .63 (moderate agreement) for women and kappa $=.51$ (weak agreement) for men.

Controlling for item content (H1b). We repeated this analysis using a more limited set of PRSPS-V items, excluding those related to the tactics of misuse of authority and enticement as there are no comparable SES-SFV items. Thus, we next compared 14 PRSPS-V items to all of the SES-SFV items. In this analysis, the SES-SFV identified 260 participants while the PRSPS- 
V identified 294. The SES-SFV identified 45 participants that the PRSPS-V did not; the PRSPSV identified 79 participants that the SES-SFV did not for a total of 124 discordant cases. Percent agreement was $81.6 \%$ (i.e., 549/673) for lifetime sexual victimization when controlling for item content. The rate of discordance was statistically significant, $\chi(1)=262.04, p<.001$, and kappa was .62 indicating moderate agreement, $\mathrm{Phi}=.38$ (medium). In this comparison, there was no difference between the PRSPS-V and the SES-SFV in detecting cases of sexual victimization for women (52.9\% vs. 51.5\%), but there was still a difference in detecting cases for men (31.2\% vs. 21.8\%), $\chi^{2}(1)=6.75, p=.009$

\section{Hypothesis 2: Discordance for specific tactics}

H2a: Verbal coercion. Overall prevalence rates for verbal coercion were $28.2 \%$ for the SES-SFV (38.4\% of women, $14.4 \%$ of men) and $42.6 \%$ for the PRSPS-V (52.3\% of women, 29.5\% of men). We compared 16-20 SES-SFV items that assessed verbal coercion victimization to 5 similar PRSPS-V items. The SES-SFV identified 190 cases while the PRSPS-V identified 287 cases. The SES-SFV identified 19 cases that PRSPS-V did not; the PRSPS-V identified 116 participants that the SES-SFV did not for a total of 135 cases discordant for verbal coercion. Percent agreement was 79.9\% (i.e., 538/673), and the rate of discordance was statistically significant, $\chi(1)=242.7, p<.001$ indicating the number of cases of sexual victimization identified by each measure was statistically different. Kappa was .57, indicating weak agreement. The effect size for this concordance was Phi $=.60$, the effect size for discordance was Phi $=.40$ (medium-large). Follow-up analyses testing for gender differences indicated that percent agreement was not significantly different for women (80.1\%) than men (80.2\%). However, kappa values did fall into different interpretative categories, kappa = .61 (moderate agreement) for women and kappa $=.44$ (weak agreement) for men. 
H2b: Alcohol or drugs. Overall prevalence rates for alcohol/drug-facilitated victimization were $13.4 \%$ for the SES-SFV (28.3\% of women, $10.7 \%$ of men) and $17.8 \%$ for the PRSPS-V (22.6\% of women, 12.1\% of men). We compared 8-10 SES-SFV items that assessed victimization via alcohol or drug intoxication to 2 similar PRSPS-V items. The SES-SFV identified 138 cases while the PRSPS-V identified 120 cases. The SES-SFV identified 48 cases that PRSPS-V did not; the PRSPS-V identified 30 participants that the SES-SFV did not for a total of 78 cases discordant for alcohol/drugs victimization. Percent agreement was $88.4 \%$ (i.e., 595/673), $\chi(1)=266.1, p<.001$, indicating the number of cases identified by the SES-SFV and the PRSPS-V were statistically different. Kappa was .63, indicating moderate agreement. The effect size for this concordance was Phi $=.63$, effect size for discordance was $=.37$ (medium). There was a significant effect of gender on discordance, $\chi(1)=9.25, p=.002$, such that percent agreement for alcohol victimization was greater for men $(92.6 \%$, kappa $=.64)$ than for women $(85.0 \%$, kappa $=.61)$.

H2c: Physical Force. Overall prevalence rates for physical force victimization were 8.9\% for the SES-SFV (20.2\% of women, $6.0 \%$ of men) and $15.2 \%$ for the PRSPS-V (19.9\% of women, 9.1\% of men). We compared 16-20 SES-SFV items that assessed victimization via physical force of threats of force to 6 similar PRSPS-V items. The SES-SFV identified 95 cases while the PRSPS-V identified 102 cases. The SES-SFV identified 35 cases that PRSPS-V did not; the PRSPS-V identified 42 participants that the SES-SFV did not for a total of 77 cases discordant for physical force victimization. Percent agreement was 88.6\% (i.e., 596/673), and the number of cases identified by the PRSPS-V and the SES-SFV were statistically different from each other, $\chi(1)=198.2, p<.001$. Kappa was .54, indicating weak agreement. The effect size for 
concordance was Phi $=.64$, the effect size for discordance was Phi $=.46$ (medium-large). There was no effect for gender.

We repeated this analysis using a limited set of PRSPS-V items that corresponded directly to SES-SFV item content (see also Table 1), specifically items 14 (physical restraint), 16 (threats of physical force), and 18 (threatened with a weapon). Prevalence rates for this limited set of physical force items were $8.2 \%$ for the SES-SFV (20.2\% of women, $6.0 \%$ of men) and 10.3\% for the PRSPS-V (14.2\% of women, $5.0 \%$ of men). Thus, we compared the same $16-20$ SES-SFV items to 3 PRSPS-V items. The SES-SFV identified 95 cases while the PRSPS-V identified 69 cases. The SES-SFV identified 40 cases that the PRSPS-V did not; the PRSPS-V identified 14 cases that the SES-SFV did not for a total of 54 discordant cases for physical force victimization. Percent agreement was 92.0\% (i.e., 619/673) which was statistically significant, $\chi(1)=272.9, p<.001, \mathrm{kappa}=.63$ (moderate), Phi $=.36$ (medium).

Hypothesis 3: Tests of order effects. We next tested whether completing the SES-SFV first (or not) affected the pattern of concordance/discordance. To do so, we compared the four possible patterns of concordance/discordance (both no, SES-SFV yes/PRSPS-V no, SES-SFV no/PRSPS-V yes, both yes) by order of administration of the SES-SFV using a chi-square of the $2 \mathrm{X} 4$ matrix. There was an effect for order of administration, $\chi(3)=11.46, p=.009$, Phi $=.13$ (small). We conducted follow-up chi-squares comparing each concordance/discordance pattern to the "both yes" group. These analyses indicated that when the SES-SFV was administered first, more participants were likely to be categorized in the SES-SFV yes/PRSPS-V no pattern than when the PRSPS-V was administered first (22 participants SES-SFV yes/PRSPS-V no vs. 8 participants SES-SFV yes/PRSPS-V no). 


\section{Discussion}

Even though rape victimization is fairly common and the source of much research, the field has struggled to accurately estimate the scope of sexual violence due to challenges in measurement. This study compared two well-used measures of sexual violence victimization to assess how differences in measurement strategy were related to differences in prevalence rates for sexual victimization in college women and men. Indeed, according to the SES-SFV, the prevalence rate of any sexual victimization for the sample would be $38.6 \%$; however, including cases identified by the PRSPS- $V$, the prevalence rate jumps to 53.2\% (14.6 percentage points). This degree of difference between the two measures was not uncommon even when considering specific tactics and controlling for item content. This effect was especially notable for men; the PRSPS-V consistently detected higher prevalence rates for men in every tactic of victimization assessed. This was true for women only when considering verbal coercion. Thus, the difference in prevalence rates between the two measures appears to be largely related to the detection of cases in men by the PRSPS-V and the inclusion of the enticement and arousal items. This is the first study to compare the SES-SFV to the PRSPS-V, document differences in sexual victimization measurement across men and women, provide kappa estimates, and control for some of the methodological issues (such as randomization of questionnaires, item content differences, response formats) raised by Strang et al. (2013).

\section{Gender Differences}

Consistent with Hypothesis 1 we found that the PRSPS-V documented higher prevalence rates for both genders. Interestingly, when controlling for item content, this effect largely disappeared for women but remained for men, suggesting a gender difference specific to the questionnaire used. Further, the difference in prevalence rates for men was fairly large; a 
difference of 32.6 vs. $21.8 \%$ (10.8 percentage points). This is consistent with prior research documenting lower validity correlations for measures of sexual victimization in men (French et al., 2017; Anderson et al., 2018a) than when the same measures are administered to women. It is possible that most sexual victimization questionnaires, developed and validated with women but subsequently adapted for other genders, are less applicable to men. It may be that the gendersensitive development and the gender-neutral wording of items of the PRSPS-V reduces heterosexism allowing for greater responses from men.

We also documented several gender differences in kappa estimates. While the differences in percent agreement were often not significant between genders, the corresponding kappa estimates were often in different interpretative categories, again indicating a greater difficulty in assessing sexual victimization in men. With the exception of alcohol, kappa estimates were consistently lower for men. Gender differences in discordance in relation to alcohol-facilitated violence were unexpected. We speculate that this finding may be related to gender differences in alcohol metabolization; men metabolize alcohol more efficiently than women do and are thus less affected by the same quantities of alcohol (Thomasson, 2002). Perhaps these differences in alcohol metabolization differentially affect men and women’s ability to accurately recall alcohol-facilitated victimization.

\section{Discordance Findings}

Consistent with Hypotheses 1, 2, and prior research, we found significant discrepancies in reported rates of sexual victimization between the two measures (Anderson et al., 2018b; Strang et al., 2013). Effect sizes of these differences were consistently in the medium-sized range, Phi $=.37-.46$. Although we would naturally expect some discrepancies between the two measures, the size of the effects documented here are still somewhat surprising. Notably, these 
differences are in spite of modifications to the questionnaires to make them more comparable and with percent agreement in ranges that would appear to be acceptable (79.9 - 92.0\%). Kappa estimates were consistent with predictions and all in the weak to moderate agreement range. Kappa estimates reflecting concordance were best for alcohol-facilitated victimization (H2b) and worst for victimization under threat of or actual physical force (H2c); estimates were also weak for verbal coercion $(\mathrm{H} 2 \mathrm{a})$. In general, kappa estimates in this range (kappa $=.54-.64)$ suggest that $29-36 \%$ of the data were reliably coded (McHugh, 2012). This is clearly below the standard kappa $\geq .80$, which would indicate $64 \%$ or more of that data is reliably coded (McHugh, 2012).

Strang and colleagues suggested that differences between the perpetration versions of these questionnaires were related to differences in item content, response formats, questionnaire structure, and the conceptualization of consent. However, we matched item content in this study as well as response formats as highlighted in prior research (DiLillo et al., 2006). This improved concordance over that reported by Strang (percent agreement of 60.7\% in Strang et al., 2013 to 82.2\% in this study for overall victimization) but did not eliminate important numerical and statistical differences in the number of cases identified by each questionnaire. Thus, the results of this study highlight the impact of item content and response format (as these improved concordance) while also pointing to other features of these questionnaires that impact victimization and perpetration prevalence rates for both men and women.

Research since Strang et al., 2013 has provided evidence for some of their hypotheses, pinpointing particular structural aspects of the questionnaires that may explain our findings. The PRSPS-V uses a simpler and non-hierarchical structure which may facilitate increased responding, especially for verbal coercion (Testa, Hoffman, Lucke \& Pagnan, 2015). In addition, the length of some SES-SFP/SES-SFV items may be problematic and require a higher reading 
level (Testa et al., 2015), which research suggests causes confusion and possibly disengagement from the questionnaire (see also, Strang \& Peterson, 2017). Participants are less careless when items are more easily interpretable (Swain et al., 2008); it may be that these specific items, rather than providing clarity, increased confusion for some participants. However, in this study it is impossible to disentangle the issue of the length of items and the structure of the questionnaires. Research from intimate partner violence literature suggests that randomizing the order of items, as the PRSPS-V does, also facilitates greater disclosure (Dietz \& Jasinski, 2007; Hamby, Sugarman, \& Boney-McCoy, 2006).

Another structural difference between the questionnaires is the inherently gendered nature of the SES-SFV. The SES-SFV items are centrally organized around describing the sexual act that the person was forced to perform. The SES-SFV therefore describes genitals and with this design feature, will retain an inherent gender difference in the number of items; people who have or have had vaginas will always complete additional items. In contrast, the PRSPS-V is rather gender neutral by describing tactics instead of sexual acts and body parts. Finally, there are established gender differences in how college students communicate their own consent and cues relied upon to ascertain consent (Jozkowski, Peterson, Sanders, Dennis \& Reece, 2013). The PRSPS-V uses a behavioral operationalization “used tactics... after you have indicated “no” to their sexual advance?” whereas the SES-SFV uses the phrase "without my consent”. It may be that young men, who rely more upon nonverbal (i.e., behavioral) signs of consent therefore endorse more PRSPS-V items. Thus, we point to item length, questionnaire structure, and conceptualization of consent as important areas for future research.

\section{Order of Administration}


Unexpectedly, we found that the order in which questionnaires were administered had an effect on patterns of concordance/discordance although the impact of this effect was small (Hypothesis 2). This finding is surprising given Anderson et al., (2018b)’s findings comparing the SES-SFV and the CTS2-SC and finding no effect for order of administration. However, they did find an order effect for the perpetration versions of these questions, suggesting that order effects may be complicated. In this study, when the SES-SFV was administered first, participants were more likely to report a pattern of “yes” responses on the SES-SFV but “no” responses on the PRSPS-V. The cause of this order effect is unclear but perhaps reflects participant fatigue.

\section{Limitations}

Discordance is only one way to assess the psychometric properties of two measures of the same construct and cannot not ultimately determine the accuracy or validity of a given measure. Thus, the findings presented in this paper should be utilized as one piece of evidence to evaluate the questionnaires at hand, with the caveat that these questionnaires were edited to test specific measurement questions and therefore our results are not a recommendation to modify these questionnaires in future non-measurement related research. Kappa estimates are sensitive to sample size (Sim \& Wright, 2005); thus, although our sample of men was relatively large, for analyses focusing on specific types of sexual victimization (e.g., physical force victimization) our subsample sizes were much smaller. We did not have adequate power to conduct concordance analyses on gender minorities, or the sexual minority subsamples, and our sample of college students was overall limited in racial and ethnic diversity. We recommend further measurement research such as this with other samples. Finally, although our changes to the PRSPS-V were to align its content with the SES-SFV and consistent with Strang et al. (2013) the validity of these items has not been studied; indeed, to our knowledge, the validity of the PRSPS- 
V items has not been examined since 2003. Thus, although the PRSPS-V appears to be a promising tool, we recommend further research on the psychometric properties of this measure.

\section{Implications for Research}

This study highlights the lack of precision in sexual victimization estimates, even when administering commonly-used questionnaires with evidence of validity. Our findings also suggest that research using the unaltered SES-SFV and PRSPS-V could underestimate sexual victimization, especially with male participants. We strongly recommend future research on sexual violence measurement, particularly focusing on how differences in questionnaire structure (e.g., the order of items), item structure (e.g., length, etc.), and the operationalization of consent impact prevalence rates and validity. PRSPS-V items are shorter and presented in a nonhierarchical (randomized) order using a behaviorally specific operationalization of consent; future research is needed that examines the extent to which each of these features impacts reporting. For example, in Strang and Peterson (2017) approximately half of the sample reported perceiving "without your consent" as a more stringent standard than "after she initially said no" suggesting there is great nuance in how consent is perceived and interpreted in questionnaires. However, is it largely unknown how changes to questionnaires (adding items, changing response formats, varying definitions of consent, etc.) may change their psychometric properties. Thus, we do not recommend that researchers wholesale implement the types of changes deployed in this study to answer measurement related research questions.

We also recommend construct validity research using interview techniques, wherein participants complete the questionnaire version and follow-up interviews about their experiences, in line with Testa et al. (2004), and Strang and Peterson (2017). Finally, we strongly suggest establishing research networks as suggested by Follingstad and Bush (2014) to conduct the 
complex and important work of measurement research to maximize limited resources and collaboratively set the research agenda for the field.

\section{Implications for Clinical Work, Prevention, and Policy}

This growing body of research suggests that using a single measure to assess sexual victimization may result in inaccurate prevalence estimates. Thus, we recommend the use of multiple questionnaires and/or follow-up interviews for those in clinical practice to assess sexual victimization histories. The rate of sexual violence victimization endured by the small population of gender minority participants in this study was $100 \%$, in line with literature suggesting the risk of sexual violence for gender minorities is very high (Stotzer, 2009); we strongly recommend future research with this community and other diverse populations. It may be that sexual and gender minorities would benefit from measures designed with their specific needs and risks in mind (Anderson, Tarasoff, VanKim, \& Flanders, 2019). Finally, the efficacy of efforts to reduce the risk of sexual violence may be inaccurately assessed, especially in under-studied and disadvantaged populations, if the assessment of prevalence rates are inaccurate. Intervention effects would be likely to be mis-estimated using measures that have poor validity and/or reliability. Thus, improving the measurement of sexual violence ultimately improves risk reduction and prevention efforts.

\section{Conclusions}

This study underscores the lack of precision in sexual victimization research, even when using common and validated measures like the Sexual Experiences Survey - Short Form Victimization and the Post-Refusal Sexual Persistence Scale - Victimization. Results highlight the need to investigate the psychometric properties of sexual victimization questionnaires in multiple populations and conduct further measurement research. 


\section{References}

Anderson, R. E., Cahill, S. P., \& Delahanty, D. L. (2018a). The psychometric properties of the Sexual Experiences Survey-Short Form Victimization (SES-SFV) and characteristics of sexual victimization experiences in college men. Psychology of Men and Masculinity, 19, 25-34. doi:10.1037/men0000073

Anderson, R. E., Cahill, S. P., \& Delahanty, D. L. (2018b). Discordance between the Sexual Experiences Surveys-Short Forms and the Revised Conflict Tactics Scales in college men. Psychology of Violence. doi:10.1037/vio0000199

Anderson, R. E., Tarasoff, L. A., VanKim, N., \& Flanders, C. (2019). Differences in rape acknowledgment and mental health outcomes across transgender, nonbinary, and cisgender bisexual youth. Journal of Interpersonal Violence, 088626051982976. doi.org/10.1177/0886260519829763

Anthony, E. R., \& Cook, S. L. (2012). Assessing the impact of gender-neutral language on disclosure of sexual violence. Psychology of Violence, 2, 297-307. doi:10.1037/a0028562

Basile, K. C., Smith, S., Breiding, M. J., Black, M. C., \& Mahendra, R. (2014). Sexual Violence Surveillance: Uniform Definitions and Recommended Data Elements, Version 2.0. Atlanta, GA. https://www.cdc.gov/violenceprevention/pdf/sv_surveillance_definitionsl-2009-a.pdf

Bouffard, L., \& Goodson, A. (2017). Sexual coercion, sexual aggression, or sexual assault: how measurement impacts our understanding of sexual violence. Journal of Aggression, Conflict and Peace Research, 9, 269-278. doi:10.1108/JACPR-05-2017-0292

Buday, S. K., \& Peterson, Z. D. (2015). Men’s and women’s interpretation and endorsement of items measuring self-reported heterosexual aggression. Journal of Sex Research, 52, 10421053. doi.org/10.1080/00224499.2014.967373 
Cascardi, M., \& Muzyczyn, B. (2016). Concordant responding on the physical assault/abuse subscales of the Revised Conflict Tactics Scales 2 and Conflict in Adolescent Dating Relationships Inventory. Psychology of Violence, 6, 303-312. doi:10.1037/a0039128

Cecil, H., \& Matson, S. C. (2006). Sexual victimization among African American adolescent females: Examination of the reliability and validity of the Sexual Experiences Survey. Journal of Interpersonal Violence, 21, 89-104. doi.org/10.1177/0886260505281606

Cook, S. L. (2002). Self-reports of sexual, physical, and nonphysical abuse perpetration. Violence Against Women, 8, 541-565. doi.org/10.1177/107780102400388425

Davis, K. C., Gilmore, A. K., Stappenbeck, C. A., Balsan, M. J., George, W. H., \& Norris, J. (2014). How to score the Sexual Experiences Survey? A Comparison of nine methods. Psychology of Violence, 4, 445-461. doi.org/10.1037/a0037494

Diamantopoulos, A., Riefler, P., \& Roth, K. P. (2008). Advancing formative measurement models. Journal of Business Research, 61, 1203-1218. doi:10.1016/j.jbusres.2008.01.009

Dietz, T. L., \& Jasinski, J. L. (2007). The effect of item order on partner violence reporting: An examination of four versions of the revised Conflict Tactics Scales. Social Science Research, 36, 353-373.

DiLillo, D., Fortier, M. A., Hayes, S. A., Trask, E., Perry, A. R., Messman-Moore, T., ... Nash, C. (2006). Retrospective assessment of childhood sexual and physical abuse: A Comparison of scaled and behaviorally specific approaches. Assessment, 13, 297-312. doi:10.1177/1073191106288391

Follingstad, D. R., \& Bush, H. M. (2014). Measurement of intimate partner violence: A model for developing the gold standard. Psychology of Violence, 4, 369-383. doi:10.1037/a0037515 
French, B. H., Suh, H. N., \& Arterberry, B. (2017). Exploratory factor analysis and psychometric properties of the Sexual Coercion Inventory. The Journal of Sex Research, 54, 962-970. doi:10.1080/00224499.2016.1235129

Hamby, S., Sugarman, D. B., \& Boney-McCoy, S. (2006). Does questionnaire format impact reported partner violence rates?: An experimental study. Violence and Victims, 21, 507-18. doi.org/10.1891/vivi.21.4.507

Hipp, T. N., \& Cook, S. L. (2017). Rape and sexual assault on campus, in diverse populations, and in the spotlight. In C. M. Renzetti, J. L. Edleson, \& R. K. Bergen (Eds.), Sourcebook on Violence Against Women (3rd ed., pp. 85-101). Thousand Oaks, CA, USA: SAGE Publications.

Johnson, S. M., Murphy, M. J., \& Gidycz, C. A. (2017). Reliability and validity of the Sexual Experiences Survey - Short Forms victimization and perpetration. Violence and Victims, 32, 78-92. doi:10.1891/0886-6708.VV-D-15-00110

Jozkowski, K. N., Peterson, Z. D., Sanders, S. A., Barbara, D., \& Michael, R. (2014). Gender differences in heterosexual college students' conceptualizations and indicators of sexual consent: Implications for contemporary sexual assault prevention education, The Journal of Sex Research, 51, 904-916. doi.org/10.1080/00224499.2013.792326

Karabatsos, G. (1997). The Sexual Experiences Survey: Interpretation and validity. Journal of Outcome Measurement, 1, 305-328.

Koss, M. P., Abbey, A., Campbell, R., Cook, S., Norris, J., Testa, M., ... White, J. (2007). Revising the SES: A Collaborative process to improve assessment of sexual aggression and victimization. Psychology of Women Quarterly, 31, 357-370. doi:10.1111/j.14716402.2007.00385.x 
Koss, M. P., \& Gidycz, C. A. (1985). Sexual Experiences Survey: Reliability and validity. Journal of Consulting and Clinical Psychology, 53, 422-423. doi.org/10.1037/0022006X.53.3.422

Koss, M. P., \& Oros, C. J. (1982). Sexual Experiences Survey: A research instrument investigating sexual aggression and victimization. Journal of Consulting and Clinical Psychology, 50, 455-457.

Krahé, B., Reimer, T., Scheinberger-Olwig, R., \& Fritsche, I. (1999). Measuring sexual aggression: The reliability of the Sexual Experiences Survey in a German sample. Journal of Interpersonal Violence, 14, 91-100.

Krahé, B., \& Vanwesenbeeck, I. (2015). Mapping an agenda for the study of youth sexual aggression in Europe: assessment, principles of good practice, and the multilevel analysis of risk factors. Journal of Sexual Aggression, 2600, 1-16.

doi:10.1080/13552600.2015.1066885

McHugh, M. L. (2012). Interrater reliability: the kappa statistic. Biochemia Medica, 22, 276282.

Moreau, C., Boucher, S., Hébert, M., \& Lemelin, J. (2014). Capturing sexual violence experiences among battered women using the Revised Sexual Experiences Survey and the Revised Conflict Tactics Scales. Archives of Sexual Behavior, 44, 223-231. doi:10.1007/s10508-014-0345-8

Muehlenhard, C. L., Peterson, Z. D., Humphreys, T. P., \& Jozkowski, K. N. (2017). Evaluating the one-in-five statistic: Women's risk of sexual assault while in college. Journal of Sex Research. Taylor \& Francis. doi:10.1080/00224499.2017.1295014 
Peterson, Z. D., Voller, E. K., Polusny, M. A., \& Murdoch, M. (2010). Prevalence and consequences of adult sexual assault of men: Review of empirical findings and state of the literature. Clinical Psychology Review, 31, 1-24. doi:10.1016/j.cpr.2010.08.006

Post, L. A., Biroscak, B. J., \& Barboza, G. (2011). Prevalence of sexual violence. In J. W. White, M. P. Koss, \& A. E. Kazdin (Eds.), Violence against women and children, Vol 1: Mapping the terrain (1st ed., pp. 101-123). Washington: American Psychological Association. doi: 10.1037/12307-005"

Sim, J., \& Wright, C. C. (2005). The kappa statistic in reliability studies: Use, interpretation, and sample size requirements. Physical Therapy, 85, 257-268.

Strang, E., Peterson, Z. D., Hill, Y. N., \& Heiman, J. R. (2013). Discrepant responding across self-report measures of men's coercive and aggressive sexual strategies. Journal of Sex Research, 50, 458-469. doi:10.1080/00224499.2011.646393

Strang, E., \& Peterson, Z. D. (2017). Unintentional misreporting on self-report measures of sexually aggressive behavior: An interview study. Journal of Sex Research, 54, 971-983. doi.org/10.1080/00224499.2017.1304519

Stotzer, R. L. (2009). Violence against transgender people: A review of United States data. Aggression and Violent Behavior, 14, 170-179. doi:10.1016/j.avb.2009.01.006

Struckman-Johnson, C., Struckman-Johnson, D., \& Anderson, P. B. (2003). Tactics of sexual coercion: When men and women won't take no for an answer. Journal of Sex Research, 40, 76-86. doi.org/10.1080/00224490309552168

Swain, S. D., Weathers, D., \& Niedrich, R. W. (2008). Assessing three sources of misresponse to reversed Likert items. Journal of Marketing Research, 45, 116-131. 
Testa, M., Hoffman, J. H., Lucke, J. F., \& Pagnan, C. E. (2015). Measuring sexual aggression perpetration in college men: A comparison of two measures. Psychology of Violence, 5, 285-293. doi.org/10.1037/a0037584

Testa, M., VanZile-Tamsen, C., Livingston, J. A., \& Koss, M. P. (2004). Assessing women’s experiences of sexual aggression using the sexual experiences survey: Evidence for validity and implications for research. Psychology of Women Quarterly, 28, 256-265. doi.org/10.1111/j.1471-6402.2004.00143.x

Teten Tharp, A., DeGue, S., Valle, L. A., Brookmeyer, K. A., Massetti, G. M., \& Matjasko, J. L. (2013). A Systematic qualitative review of risk and protective factors for sexual violence perpetration. Trauma, Violence, and Abuse, 14. doi.org/10.1177/1524838012470031

Thomasson, H. R. (2002). Gender differences in alcohol metabolism. In Recent developments in alcoholism (pp. 163-179). Springer, Boston, MA.

Turchik, J. A., \& Hassija, C. M. (2014). Female sexual victimization among college students: Assault severity, health risk behaviors, and sexual functioning. Journal of Interpersonal Violence, 29, 2439-2457. doi.org/10.1177/088626051352 